\title{
Frontières
}

\section{Étude des facteurs d'ajustement au deuil après un suicide et après un décès soudain non intentionnel}

\section{Chantal Bournival, Monique Séguin et Marc-Simon Drouin}

Volume 24, numéro 1-2, automne 2011, printemps 2012

URI : https://id.erudit.org/iderudit/1013085ar

DOI : https://doi.org/10.7202/1013085ar

Aller au sommaire du numéro

Éditeur(s)

Université du Québec à Montréal

ISSN

1916-0976 (numérique)

Découvrir la revue

Citer cet article

Bournival, C., Séguin, M. \& Drouin, M.-S. (2011). Étude des facteurs d'ajustement au deuil après un suicide et après un décès soudain non intentionnel. Frontières, 24(1-2), 55-62. https://doi.org/10.7202/1013085ar
Résumé de l'article

La question de savoir si le deuil après suicide est plus difficile qu'une autre forme de deuil est encore débattue dans la littérature contemporaine. Cependant, outre la nature du décès, d'autres variables peuvent expliquer la difficulté du processus d'ajustement au deuil. Parmi ces variables, il y a la satisfaction de couple, la satisfaction de vie et l'attachement. Cette étude a comparé des femmes veuves endeuillées après un suicide $(n=22)$ et des femmes veuves endeuillées à la suite d'un décès non intentionnel $(n=24)$. Les résultats indiquent que les femmes endeuillées par le suicide d'un proche, comparativement à celles ayant vécu un autre type de deuil à la suite d'un décès non intentionnel, rapportaient davantage de détresse psychologique et une plus grande insatisfaction face à la vie et au couple. En revanche, la détresse ressentie n'est pas associée à la nature du décès, mais le niveau de satisfaction de vie et de couple serait davantage lié à la difficulté du deuil vécu. 


\section{Résumé}

La question de savoir si le deuil après suicide est plus difficile qu'une autre forme de deuil est encore débattue dans la littérature contemporaine. Cependant, outre la nature du décès, d'autres variables peuvent expliquer la difficulté du processus d'ajustement au deuil. Parmi ces variables, il y a la satisfaction de couple, la satisfaction de vie et l'attachement. Cette étude a comparé des femmes veuves endeuillées après un suicide $(n=22)$ et des femmes veuves endeuillées à la suite d'un décès non intentionnel $(n=24)$. Les résultats indiquent que les femmes endeuillées par le suicide d'un proche, comparativement à celles ayant vécu un autre type de deuil à la suite d'un décès non intentionnel, rapportaient davantage de détresse psychologique et une plus grande insatisfaction face à la vie et au couple. En revanche, la détresse ressentie n'est pas associée à la nature du décès, mais le niveau de satisfaction de vie et de couple serait davantage lié à la difficulté du deuil vécu.

Mots clés: deuil - suicide - veuves satisfaction de couple - satisfaction de vie - attachement.

\section{Abstract}

Whether mourning after a suicide is more complex than other types of mourning remains a debated question in contemporary literature. However, apart from the nature of the death, other variables can also explain the complexity of the mourning-adjustment process. Among these variables, there is couple satisfaction, life satisfaction and attachment. This study has compared widows in mourning after a suicide ( $n=22)$ and widows in mourning following a non-intentional death $(n=24)$. The results indicate that women mourning in relation to suicide, as opposed to those having experienced mourning due to other types of non-intentional death, have reported more psychological distress and a greater dissatisfaction towards life and the couple. On the other hand, the distress experienced is not associated with the nature of the death, but life and couple satisfaction would seem to be associated more importantly with the mourning difficulty.

Keywords: mourning - suicide - widows - couple satisfaction - life satisfaction attachment.

\section{Étude des facteurs d'ajustement au deuil après un suicide et après un décès soudain non intentionnel}

Chantal Bournival, Psy. D.,

Groupe McGill d'études sur le suicide, Hôpital Douglas.

Monique Séguin, Ph. D.,

Université du Québec en Outaouais,

Département de psychoéducation et de psychologie.

Marc-Simon Drouin, Ph. D.,
Université du Québec à Montréal
Département de psychologie.

Le décès d'un être cher est un événement difficile, auquel la majorité des gens seront confrontés au cours de leur vie. Certains facteurs pourraient en compliquer le processus d'ajustement, dont le type de décès. Parmi l'impact des différents types de décès, le deuil par suicide a été étudié par de nombreux auteurs en raison de son caractère particulier. D'ailleurs, les répercussions du deuil après suicide interpellent beaucoup les chercheurs et les résultats des études scientifiques sont controversés. Plusieurs auteurs ont rapporté que le deuil par suicide était plus complexe que les autres types de deuil soit au niveau de la durée du processus de deuil, de l'intensité de la détresse psychologique, etc. (Jordan, 2008; Hawton, Simkin et Rees, 2008; De Groot, Keijser et Neeleman, 2006 ; Mitchell et al., 2006; Currier, Holland et Neimeyer, 2006; Requarth, 2006; Cvinar, 2005; Winter, Brockmann et Hegerl, 2005 ; Zhang, Tong et Zhou, 2005; Jordan et McMenamy, 2004; Mitchell et al., 2003 ; Domingos et Maluf, 2003; Sethi et Bhargava, 2003 ; Snow et McHugh, 2002). En revanche, le fait que la majorité de ces études ont été menées en absence de groupe de comparaison (Jordan, 2008; Hawton, Simkin et Rees, 2008; Mitchell et al., 2006; Currier, Holland et Neimeyer, 2006; Requarth, 2006; Cvinar, 2005; Winter, Brockmann et Hegerl, 2005; Jordan et McMenamy, 2004; Mitchell et al., 2003; Domingos et Maluf, 2003 ; Snow et McHugh, 2002) limite la portée des résultats, car ils ne permettent pas de distinguer les caractéristiques propres au deuil par suicide. D'autre part, certaines études comparent (quant à l'ajustement social, la présence de symptômes psychiatriques, la présence 
d'un trouble de l'humeur) des personnes endeuillées à des personnes non endeuillées (Zhang, Tong et Zhou, 2005 ; Sethi et Bhargava, 2003).

Un autre courant de données scientifiques rapportent peu ou pas de différence significative dans des études comparant des personnes endeuillées par suicide à des personnes endeuillées par d'autres types de décès quant à la difficulté de leur deuil (Sveen et Walby, 2008; Melhem et al., 2007 ; Brown et al., 2007 ; Fitzgerald, 2005; Rengifo, 2004; Dyregrov, Nordanger et Dyregrov, 2003; Murphy et al., 2003; Murphy, Johnson et Lohan, 2003 ; Murphy et al., 2002; Liebscher, 2001; Cerel et al., 2000 ; Kitson, 2000). Bien que plusieurs de ces études aient des groupes de comparaison (Melhem et al., 2007; Brown et al., 2007 ; Fitzgerald, 2005 ; Rengifo, 2004 ; Dyregrov, Nordanger et Dyregrov, 2003; Murphy et al., 2003; Murphy, Johnson et Lohan, 2003; Murphy et al., 2002; Liebscher, 2001; Kitson, 2000 ; Cerel et al., 2000), plusieurs d'entre elles combinent les types de deuil ayant eu lieu subitement (suicide, homicide, accident) (Rengifo, 2004; Murphy et al., 2002; Liebscher, 2001; Kitson, 2000) en un seul groupe, malgré les circonstances différentes dans l'intentionnalité, et comparent ce groupe à un groupe de personnes ayant un deuil prévu, ce qui rend d'autant plus difficile l'interprétation de ce qui est attribuable à la nature spécifique du décès.

D'autre part, quelques auteurs ont suggéré que les différences dans le processus de deuil pourraient être expliquées par des variables présentes antérieurement au décès plutôt que par la nature du décès (Séguin et al., 2009; Tall et al., 2008; Bonnanno, Boerner et Wortman, 2008). Lorsque la perte est celle d'un conjoint, certaines variables en lien avec la situation conjugale avant le décès du partenaire pourraient avoir une influence sur le processus de deuil. L'intensité du bouleversement et la présence de diverses émotions à la suite du décès (culpabilité, honte, tristesse, etc.) pourraient être influencées par diverses variables en lien avec la relation de couple. Parmi ces variables, nous retrouvons la qualité de la relation conjugale avant le décès du conjoint (Waskowic et Chartier, 2003; Colin, 1996; Wortman et Silver, 1990; 1989; Gass, 1989; Bozzini et Tessier, 1989; Parkes et Weiss, 1983; Worden, 1982; Kalish, 1981; Glick, Parkes et Weiss, 1974), la satisfaction de vie (Schaefer et Moos, 2001; Stein et al., 1997) et le type d'attachement (Stroebe, Schut et Stroebe, 2005 ; Fraley et Bonanno, 2004; Waskowic et Chartier, 2003; Van Doorn et al., 2002; Field et Sundin, 2001; Jacobs et Prigerson,
1998). Bien que ces variables aient démontré un effet spécifique sur le processus de deuil en général, à notre connaissance, aucune étude n'a mis en relation la qualité de la vie conjugale et la nature du décès.

LORSQUE LA PERTE

EST CELLE D'UN CONJOINT, CERTAINES VARIABLES

EN LIEN AVEC LA SITUATION

CONJUGALE AVANT LE DÉCÈS

DU PARTENAIRE POURRAIENT

AVOIR UNE INFLUENCE SUR

LE PROCESSUS DE DEUIL.

L'INTENSITÉ DU BOULEVERSEMENT

ET LA PRÉSENCE DE DIVERSES

ÉMOTIONS À LA SUITE DU DÉCÈS

(CULPABILITÉ, HONTE,

TRISTESSE, ETC.) POURRAIENT

ÊTRE INFLUENCÉES PAR DIVERSES

VARIABLES EN LIEN

AVEC LA RELATION DE COUPLE.

Nous souhaitons vérifier si des variables comme la qualité de la vie conjugale ou la satisfaction de vie peuvent avoir des répercussions sur le deuil, au-delà de la nature potentiellement traumatique du décès d'un conjoint, provoqué par un geste délibéré ou par mort non intentionnelle. Dans ce contexte, les veuves endeuillées se révèlent une population d'intérêt étant donné que $75 \%$ des personnes qui se suicident au Québec sont des hommes (MSSS, 2007).

L'objectif de cette étude est de vérifier si les veuves endeuillées par un suicide ont un processus de deuil plus difficile que les veuves endeuillées après un décès non intentionnel. Nous comparerons les veuves endeuillées après un suicide aux veuves endeuillées par un décès non intentionnel sur les variables actuelles et passées. Un deuxième objectif sera d'examiner si la qualité de la relation conjugale, la satisfaction de vie et le type d'attachement avant le décès du conjoint contribuent à expliquer la différence quant à l'issue du deuil. Pour ce faire, nous comparerons parmi les deux groupes, les femmes ayant un deuil difficile aux femmes ayant un deuil normal et nous tenterons d'examiner les variables qui contribuent à augmenter l'intensité du deuil.

\section{MÉTHODOLOGIE}

\section{PARTICIPANTES}

Quarante-six participantes endeuillées subitement ont été recrutées entre le 10 août 2007 et le 10 février 2009, à travers le Québec, formant deux groupes dont un premier groupe de femmes endeuillées après un suicide $(\mathrm{N}=22)$ et un deuxième groupe de femmes endeuillées suite à un décès non intentionnel $(\mathrm{N}=24)$. Les critères d'inclusion étaient les suivants: $a$ ) être une femme âgée de 40 ans et plus; b) avoir perdu son conjoint au cours des cinq dernières années; $c$ ) avoir perdu un conjoint subitement soit par suicide ou par mort non intentionnelle (crise cardiaque, accident, maladie très brève, etc.); $d$ ) avoir été en relation avec le conjoint pendant plus de dix ans.

\section{RECRUTEMENT}

Le recrutement a été réalisé en utilisant différentes stratégies. Le recrutement s'est principalement effectué à travers le Groupe McGill d'études sur le suicide, qui réalise des études sur le suicide et le deuil après suicide depuis plus de quinze ans. Cette équipe a un lien privilégié avec le Bureau du coroner du Québec et offre une aide précieuse dans le processus de recrutement. Généralement, des lettres envoyées, signées par le Bureau du coroner en chef du Québec, présentent l'étude en cours et invitent la personne à contacter l'équipe de recherche si elle trouve quelque intérêt à sa participation. Ainsi, 160 lettres ont été envoyées à des femmes endeuillées par suicide $(\mathrm{N}=81)$ ou par mort non intentionnelle $(\mathrm{N}=79)$.

Une autre stratégie de recrutement consistait à solliciter des personnes ayant participé à des études antérieures du Groupe McGill d'études sur le suicide et qui avaient donné leur accord pour un deuxième contact en vue de participer à une autre étude. Nous avons également placé deux annonces dans le journal local, une dans le Journal de l'Association des veuves de Montréal et une annonce avait été faite aux bureaux de Suicide Action Montréal. Enfin, quelques participantes ont été recrutées par le bouche à oreille.

\section{INSTRUMENTS DE MESURE}

Variables sociodémographiques

Les données sociodémographiques ont été recueillies à l'aide d'un questionnaire 
composé de 15 questions portant sur l'âge, l'origine ethnique, la religion, le nombre d'années en couple, le nombre de mois depuis le décès, le nombre de fois ayant été mariées ou en union de fait, le nombre d'enfants, le niveau de scolarisation, l'occupation actuelle, le poste professionnel le plus important occupé au cours de la vie, la principale source de revenu, le revenu total, le nombre de personnes demeurant avec la répondante et le type de décès du conjoint.

\section{Variables psychologiques}

L'intensité du deuil a été évaluée au moyen du Grief Experience Questionnaire (GEQ) (Barrett et Scott, 1989; Vallerand, 1989). Ce questionnaire est composé de 55 items mesurant les réactions de deuil. Un score total supérieur à 149 indique un deuil difficile. Une retraduction de ce questionnaire fondée sur les recommandations de Vallerand (1989) a été effectuée. Les qualités psychométriques de la traduction française du GEQ a permis d'identifier un excellent niveau de validité interne (validité interne de la version retraduite: 0,96). Ce questionnaire a été sélectionné en raison de ses qualités psychométriques. Les questions se rapportaient à la première année suivant le décès du conjoint.

La détresse psychologique lors du deuil a été évaluée à l'aide du questionnaire de l'indice de détresse psychologique (IDPESQ-14) (Préville et al., 1992; Ilfeld, 1976). Ce questionnaire est composé de 14 questions portant sur la détresse psychologique. Les questions regroupent le facteur d'anxiété, le facteur dépressif, le facteur d'irritabilité ainsi que le facteur de problèmes cognitifs. Un score total supérieur à 20 révèle une détresse cliniquement significative. La consistance interne de Cronbach de l'échelle globale est de 0,89 . Ce questionnaire a été sélectionné en raison de sa facilité d'application. Les questions se référaient à la première année suivant le décès du conjoint.

La satisfaction conjugale a été évaluée d'une manière rétrospective à l'aide de l'échelle de l'ajustement dyadique (DAS) (Baillargeon, Dubois et Marineau, 1988; Spanier, 1976). Ce questionnaire est composé de 32 items qui vérifient la qualité de l'ajustement marital et des relations similaires. Il mesure quatre dimensions $\mathrm{du}$ fonctionnement conjugal: le consensus dyadique, l'expression affective, la cohésion dyadique et la satisfaction dyadique. Un score total inférieur à 100 est signe d'une insatisfaction conjugale. Selon les études, la fidélité de l'instrument varie entre 0,91 et 0,96 (coefficients alpha). Plusieurs études ont éprouvées la fidélité et la validité de la version française de cette échelle (Sabourin et al., 1990; Sabourin et al., 1988). Ce questionnaire a été sélectionné en raison de ses qualités psychométriques.

La satisfaction de vie a été étudiée à l'aide de l'échelle de satisfaction de vivre (Blais et al., 1989; Diener, Emmons, Larsen et Griffin, 1985) qui comprend cinq items permettant une évaluation globale de la satisfaction de sa propre vie. Le questionnaire est composé d'une échelle de 1 (fortement en désaccord) à 7 (fortement d'accord). Ce questionnaire est axé sur la composante cognitive du bonheur. Un score total inférieur à 20 correspond à une insatisfaction de la vie. Cinq études effectuées par Blais et ses collègues (1989) ont confirmé sa validité ainsi que sa fidélité. L'alpha de Cronbach varie entre 0,80 et 0,84 selon les groupes étudiés. Ce questionnaire a été choisi pour ses qualités psychométriques et son temps de passation très limité.

L'échelle d'affect positif et d'affect négatif (PANAS) (Bouffard, Bastin et Lapierre, 1997; Watson et al., 1988) a également été utilisée afin d'évaluer la variable de la satisfaction de vie, mais selon une composante affective. Ce questionnaire comprend 20 adjectifs, 10 relatifs aux affects positifs et 10 relatifs aux affects négatifs. La participante est invitée à répondre à ces questions selon ses affects avant le décès de son conjoint. Il s'agit de différentes émotions proposées et la participante indique à quel point elle éprouve chacune de ces émotions de 1 (pas du tout) à 5 (extrêmement). Les affects positifs et négatifs sont deux facteurs indépendants faiblement corrélés. Lorsque la participante a un score élevé d'affect positif, ceci correspond à un état de grande énergie et d'engagement plaisant, alors qu'un score faible d'affect positif correspond à un état de tristesse et de léthargie. Lorsqu'il y a un score élevé d'affect négatif, cela correspond à un état de détresse psychologique composée d'une panoplie d'émotions déplaisantes, tandis qu'un score faible d'affect négatif correspond à un état de calme et de sérénité. Les participantes qui présentaient des scores plus élevés sur l'échelle des affects négatifs que sur l'échelle d'affects positifs étaient considérées comme ayant plus d'affects négatifs en regard de leur vie. La consistance interne (alpha) est de 0,90 pour les items positifs et de 0,77 pour les items négatifs; ce dernier résultat est moins satisfaisant que celui de l'échelle originale. Cet instrument a été sélectionné pour la valeur de l'instrument original et de sa facilité d'application.

Le type d'attachement a été évalué à l'aide du Questionnaire de l'attachement chez l'adulte (QAA) (Bouthiller et al.,
1996; Simpson, 1990). Le questionnaire est composé de 13 items, dont les cinq premiers ont trait au style d'attachement sécuritaire, les quatre suivants au style d'attachement évitant et les quatre derniers au style d'attachement anxieux/ ambivalent. Les résultats varient entre 1 et 7, 1 représentant un niveau de désaccord élevé avec l'énoncé. Les scores aux différentes échelles ont été pondérés en fonction du nombre d'items. Les participantes qui présentaient des scores plus élevés sur l'échelle d'attachement sécuritaire que sur les autres types d'attachement étaient considérées comme ayant un attachement sécuritaire, alors que des scores plus élevés sur les autres types d'attachement signifiaient un attachement insécurisant. Les indices de validité concomitante, la validité factorielle, la cohérence interne et la stabilité interne de la version française et anglaise sont comparables. La cohérence interne de la version française évaluée à l'aide du coefficient de Cronbach est de 0,77 pour l'échelle d'évitement et de 0,64 pour l'échelle d'anxiété. Enfin, les coefficients de corrélation test-retest varient entre 0,37 et 0,71 et sont tous significatifs $(p<0,01)$.

\section{PROCÉDURE}

Les personnes manifestant leur intérêt à participer étaient tout d'abord contactées par la chercheuse principale, afin qu'elle leur explique les buts de la recherche, le protocole, les procédures, la confidentialité, etc. Ensuite, si la personne acceptait de participer, un rendez-vous téléphonique ou en face à face était fixé, à un moment et à un lieu (l'hôpital Douglas ou au domicile de la participante) à la convenance de la participante pour répondre au questionnaire. Les questionnaires ont tous été administrés par la chercheuse principale. Une seule rencontre d'une durée moyenne de 90 minutes était nécessaire. Un formulaire de consentement a été signé avant le début de l'entretien et cette étude a reçu l'aval du comité éthique de l'Université du Québec à Montréal.

\section{ANALYSES STATISTIQUES}

Des analyses descriptives ont été effectuées sur les variables obtenues au questionnaire sociodémographique des répondantes (l'âge des répondantes, le nombre d'années en couple, le nombre de mois de veuvage, etc.). Pour vérifier si ces caractéristiques différaient en fonction du type de deuil (suicide $v s$ non intentionnel) et de la difficulté du deuil (difficile vs sain), des ANOVAS simples pour les variables en continu et des khi carrés pour les variables catégorielles ont été menées. 
Afin de vérifier l'effet de la nature du décès sur différents aspects psychologiques des répondantes, des analyses de khi carrés ont été effectuées sur les variables des participantes rencontrant (ou non) les seuils cliniques en ce qui concerne l'expérience de deuil, la détresse psychologique, l'attachement à l'âge adulte, les affects positifs et négatifs ainsi que la satisfaction conjugale et de vie.

De plus, afin de vérifier si la nature du décès était la variable pouvant le mieux expliquer les conséquences du deuil ou si des variables antérieures au deuil peuvent mieux expliquer les suites du deuil, nous avons comparé les groupes selon la difficulté à vivre le deuil et tenté d'examiner quelles étaient les variables pouvant distinguer ces groupes. La difficulté après un deuil a été déterminée à l'aide du Grief Experience Questionnaire. Les participantes ayant obtenu des scores d'un écart type au-dessus de la moyenne (score > 149) sur ce questionnaire ont été identifiées comme faisant partie des personnes ayant un deuil difficile $(\mathrm{N}=8)$, alors que les autres participantes ont été considérées comme vivant un deuil sain $(\mathrm{N}=38)$. Afin de vérifier l'effet de la difficulté à vivre le décès sur différents aspects psychologiques des répondantes, des analyses de khi carrés ont été effectuées sur les variables des participantes rencontrant (ou non) les seuils cliniques en ce qui concerne la détresse psychologique, l'attachement à l'âge adulte, les affects positifs et négatifs ainsi que la satisfaction conjugale et de vie.

\section{RÉSULTATS}

\section{PROFIL SOCIODÉMOGRAPHIQUE}

DES PARTICIPANTES SELON LE TYPE

DE DEUIL

Les participantes présentent un profil semblable quant au nombre d'années de relation avec leur conjoint, le nombre de mois de veuvage, le nombre d'enfants, le nombre de personnes habitant avec la personne endeuillée, le niveau de scolarité, l'occupation et le revenu (voir tableau 1). Elles diffèrent cependant sur le plan de l'âge. En effet, les femmes endeuillées après un décès non intentionnel $(\mathrm{M}=65,13$; $\mathrm{ET}=12,76 ; \mathrm{p}<0.05)$ étaient significativement plus âgées que les femmes endeuillées après un décès par suicide $(M=58,77$; $\mathrm{ET}=8,05 ; \mathrm{p}<0,05)$.

\section{DONNÉES CLINIQUES SELON}

\section{LE TYPE DE DEUIL}

Les répondantes endeuillées après un décès par suicide (voir tableau 2) étaient plus nombreuses que les répondantes endeuillées après un décès non intentionnel à présenter une détresse psychologique

TABLEAU 1

LE PROFIL SOCIODÉMOGRAPHIQUE DES PARTICIPANTES SELON LE TYPE DE DEUIL

\begin{tabular}{|c|c|c|c|}
\hline & $\begin{array}{c}\text { Groupe } \\
\text { de participantes } \\
\text { endeuillées } \\
\text { par suicide } \\
(n=22)\end{array}$ & $\begin{array}{c}\text { Groupe } \\
\text { de participantes } \\
\text { endeuillées par } \\
\text { décès soudain non } \\
\text { intentionnel } \\
(n=24)\end{array}$ & $\mathrm{P}$ \\
\hline \multicolumn{4}{|l|}{ Mesures } \\
\hline Âge du répondan̄t $\bar{x}(E t)^{*}$ & $58,77(8,05)$ & $65,13(12,76)$ & 0,048 \\
\hline $\mathrm{N}^{\text {bre }}$ années en couple $\overline{\mathrm{x}}(\mathrm{Et})$ & $30,64(9,86)$ & $37,33(15,45)$ & 0,085 \\
\hline $\mathrm{N}^{\text {bre }}$ mois veuve $\overline{\mathrm{x}}(\mathrm{Et})$ & $24,59(20,25)$ & $32,33(21,84)$ & 0,220 \\
\hline $\mathrm{N}^{\text {bre }}$ enfants bio $\overline{\mathrm{x}}(\mathrm{Et})^{*}$ & $1,59(1,10)$ & $2,50(1,62)$ & 0,032 \\
\hline $\mathrm{N}^{\text {bre }}$ de personnes habitant & $1,41(1,01)$ & $1,29(0,81)$ & 0,663 \\
\hline \multicolumn{4}{|l|}{ Scolarité du répondant $n(\%)$} \\
\hline Niveau primaire & $6(27,3)$ & $4(17,4)$ & \multirow[t]{4}{*}{0,864} \\
\hline Niveau secondaire & $6(27,3)$ & $4(17,4)$ & \\
\hline $\begin{array}{l}\text { Niveau collégial } \\
\text { ou professionnel }\end{array}$ & $4(18,2)$ & $8(34,8)$ & \\
\hline Niveau universitaire & $6(27,3)$ & $7(30,4)$ & \\
\hline \multicolumn{4}{|c|}{ Occupation du répondant $n(\%)$} \\
\hline Travailleur & $12(54,5)$ & $9(45,7)$ & \multirow[t]{3}{*}{0,436} \\
\hline Retraité & $7(31,8)$ & $12(50,0)$ & \\
\hline Sans emploi & $3(13,6)$ & $3(12,5)$ & \\
\hline \multicolumn{4}{|l|}{ Revenu du répondant $n(\%)$} \\
\hline Moins de $30000 \$$ & $9(47,4)$ & $11(50,0)$ & \multirow[t]{3}{*}{0,547} \\
\hline 30000 à $59999 \$$ & $4(21,1)$ & $7(31,8)$ & \\
\hline $60000 \$$ et plus & $6(31,6)$ & $4(18,2)$ & \\
\hline
\end{tabular}

$* \mathrm{p}<0,05 * \mathrm{p} p<0,01 * * * \mathrm{p}<0,001$

cliniquement importante $\left(\chi^{2}(1)=4,84\right.$; $\mathrm{p}<0,05)$. De plus, antérieurement au décès, celles-ci étaient plus insatisfaites de leur vie $\left(\chi^{2}(1)=7,62 ; \mathrm{p}<0,01\right)$, et de leur couple $\left(\chi^{2}(1)=10,98 ; \mathrm{p}<0,001\right)$ que les personnes du groupe de comparaison. Aucune autre différence significative n'a été observée.

Un deuxième niveau d'analyse a été fait, car nous observons que parmi les femmes qui souffrent d'un deuil difficile, il y a un plus grand nombre qui sont endeuillés par un suicide $(n=6)$ que par un décès non intentionnel $(n=2)$, mais que les participantes présentant un deuil sain se distribuent similairement qu'elles soient endeuillées par un suicide $(n=16)$ ou par un décès non intentionnel $(n=22)$. Ainsi nous avons créé deux groupes de femmes sur la base des difficultés du deuil, afin d'évaluer si la nature du décès expliquerait encore la difficulté du deuil malgré ce nouveau regroupement ou si d'autres variables (satisfaction conjugale, satisfaction de vie et type d'attachement) expliqueraient mieux la difficulté du deuil.
Nous avons donc poursuivi nos analyses en reconstituant les groupes et en créant alors un groupe de personnes ayant un deuil sain et un groupe de personnes ayant un deuil difficile.

\section{PROFIL SOCIODÉMOGRAPHIQUE \\ DES PARTICIPANTES SELON \\ LA DIFFICULTÉ DU DEUIL}

Les participantes du groupe de deuil sain $(\mathrm{M}=36,26 ; \mathrm{ET}=12,73 ; \mathrm{p}<0,05)$ étaient en couple depuis plus longtemps que les participantes du groupe de deuil difficile $(\mathrm{M}=24,00, \mathrm{ET}=12,31 ; \mathrm{p}<0,05)$. De plus, les participantes du groupe de deuil sain étaient plus nombreuses à être à la retraite, tandis que les celles du groupe de deuil difficile étaient plutôt sans emploi.

\section{DONNÉES CLINIQUES SELON \\ LA DIFFICULTÉ DU DEUIL}

Nous avons remarqué que les participantes souffrant de deuil difficile sont plus nombreuses, antérieurement au décès, à avoir été insatisfaites de leur vie $\left(\chi^{2}(1)=14,90 ; p<0,001\right)$ et de leur couple 
TABLEAU 2

LE PROFIL PSYCHOLOGIQUE DES PARTICIPANTES SELON LE TYPE DE DEUIL (N ET \%)

\begin{tabular}{|c|c|c|c|}
\hline & $\begin{array}{c}\text { Groupe } \\
\text { de participantes } \\
\text { endeuillées } \\
\text { par suicide } \\
(n=22)\end{array}$ & $\begin{array}{c}\text { Groupe } \\
\text { de participantes } \\
\text { endeuillées } \\
\text { de façon non } \\
\text { intentionnelle } \\
(n=24)\end{array}$ & $\mathrm{P}$ \\
\hline \multicolumn{4}{|l|}{ Variables } \\
\hline \multicolumn{4}{|l|}{ Variables antérieures au décès } \\
\hline Insatisfaction conjugale ${ }^{* * * *}$ & $13(59,1)$ & $3(12,5)$ & 0,001 \\
\hline Insatisfaction de vie ${ }^{* * *}$ & $11(50,0)$ & $3(12,5)$ & 0,006 \\
\hline $\begin{array}{l}\text { Affects négatifs élevés } \\
\text { en regard de la vie }\end{array}$ & $5(22,7)$ & $2(8,3)$ & 0,175 \\
\hline $\begin{array}{l}\text { Attachement de type } \\
\text { sécuritaire }\end{array}$ & $15(68,2)$ & $20(83,3)$ & 0,229 \\
\hline \multicolumn{4}{|l|}{ Variables actuelles } \\
\hline $\begin{array}{l}\text { Indice de détresse } \\
\text { psychologique élevé** }\end{array}$ & $21(95,5)$ & $17(70,8)$ & 0,028 \\
\hline Expérience de deuil difficile & $6(27,3)$ & $2(8,3)$ & 0,090 \\
\hline
\end{tabular}

TABLEAU 3

LE PROFIL PSYCHOLOGIQUE DES PARTICIPANTES SELON LA DIFFICULTÉ DU DEUIL (N ET \%)
Deuil sain
Deuil difficile
$(n=38)$
$(n=8)$
$\mathrm{P}$

\begin{tabular}{|c|c|c|c|}
\hline \multicolumn{4}{|l|}{ Variables } \\
\hline \multicolumn{4}{|c|}{ Variables antérieures au décès } \\
\hline Insatisfaction conjugale ${ }^{* * *}$ & $10(26,3)$ & $6(75,0)$ & 0,009 \\
\hline Insatisfaction de vie ${ }^{* * * *}$ & $7(18,4)$ & $7(87,5)$ & 0,000 \\
\hline $\begin{array}{l}\text { Affects négatifs élevés } \\
\text { en regard de la vie }\end{array}$ & $5(13,2)$ & $2(25,0)$ & 0,397 \\
\hline $\begin{array}{l}\text { Attachement de type } \\
\text { sécuritaire }\end{array}$ & $30(78,9)$ & $5(62,5)$ & 0,322 \\
\hline \multicolumn{4}{|l|}{ Variables actuelles } \\
\hline $\begin{array}{l}\text { Indice de détresse } \\
\text { psychologique élevé }\end{array}$ & $30(78,9)$ & $8(100,0)$ & 0,153 \\
\hline
\end{tabular}

$* \mathrm{p}<0,05 * \mathrm{p}<0,01 * * \mathrm{p}<0,001$

$\left(\chi^{2}(1)=6,91 ; p<0,01\right)$ comparativement aux femmes ayant un deuil sain (voir tableau 3). Les groupes ne différaient sur aucune autre des caractéristiques mesurées.

\section{DISCUSSION}

Cette étude a permis de constater que les veuves endeuillées par un suicide, comparativement à celles ayant vécu d'autres types de deuil après un décès non intentionnel, rapportaient une plus grande détresse psychologique. Celles-ci présentaient également des différences sur le plan des caractéristiques présentes nel, en raison du niveau de détresse plus élevé chez les personnes endeuillées par un suicide. Toutefois, des analyses supplémentaires ont permis d'affiner notre compréhension concernant le rôle de la nature du deuil dans ce processus d'ajustement. En effet, la redistribution des groupes en un groupe de deuil sain et en un groupe de deuil difficile a démontré que le

DE FAIT,

LE DÉCÈS DU CONJOINT, QU'IL SOIT PAR SUICIDE

OU PAR ACCIDENT,

PEUT AMENER CERTAINS

ENDEUILLÉS À MODULER

LEURS RÉPONSES APRÈS COUP,

SOIT EN CRÉANT UN BIAIS POSITIF

OU NÉGATIF LORSQUE

LES PARTICIPANTS DÉCRIVENT

LEUR VIE DE COUPLE

AVANT LE DÉCÈS.

groupe de deuil sain était composé à la fois de personnes endeuillées par un suicide et endeuillées par un décès non intentionnel. Le groupe de deuil difficile était toutefois composé d'une plus grande proportion de personnes endeuillées par un suicide $(n=6)$ que de personnes endeuillées par un décès non intentionnel $(n=2)$, ce qui nous permet d'observer qu'un sous-groupe de personnes endeuillées par un suicide vivent un deuil plus difficile, de la même manière qu'un sous-groupe de personnes en deuil consécutif à un accident vivent un deuil plus difficile.

Si les personnes vivant un deuil difficile sont plus nombreuses à se dire insatisfaites de leur vie antérieure au décès, pourquoi y a-t-il plus de femmes insatisfaites chez les veuves en deuil après un suicide? Plusieurs explications pourraient soutenir ces résultats. Entre autres, les études démontrent que les personnes décédées après suicide, dont $75 \%$ sont des hommes adultes (MSSS, 2007), avaient éprouvé, dans l'année précédant leur suicide, des troubles de l'humeur dans une proportion de $60 \%$ (Cavanagh et al., 2003; Kim et al., 2003), des troubles liés à une substance dans une proportion de $59 \%$ (Séguin et al., 
2006) et de façon comorbide un trouble de santé mentale et d'abus de substances dans une proportion de $42 \%$ (Séguin et al., 2006). Il serait donc possible que la présence d'une psychopathologie chez le conjoint suicidé ait occasionné une détresse dans l'univers immédiat de la conjointe. Par conséquent, ceci peut avoir augmenté le niveau d'insatisfaction de vie chez ce sous-groupe de femmes endeuillées par un suicide. De plus, bien que les troubles dépressifs et les troubles d'abus de substances ou d'alcool soient associés à plus de 90\% des suicides (Cheng et al., 2000 ; Rich et al., 1988; Brent et al., 1987; Rich et al., 1986; Barraclough et al., 1974), il y a une proportion de gens qui se sont suicidés qui n'avaient aucun trouble de santé mentale. Les femmes endeuillées par un suicide qui ont un deuil sain peuvent avoir été les conjointes de ces personnes n'ayant eu aucun trouble de santé mentale et avoir vécu alors une moindre lourdeur liée à l'état psychologique du conjoint, donc possiblement un niveau de satisfaction de vie plus élevé. Il serait intéressant de mesurer cet aspect dans de futures études afin d'élucider cette hypothèse.

Nos résultats indiquent que les personnes endeuillées par un suicide sont moins satisfaites au point de vue conjugal. Encore ici, il est probable que le fardeau issu de l'état psychopathologique du conjoint ait diminué la satisfaction conjugale. En effet, une relation de couple satisfaisante est plus susceptible de générer un sentiment de paix et d'accomplissement lors du deuil et par conséquent faciliter son processus (Colin, 1996; Bozzini et Tessier, 1989). Une relation conflictuelle ou ambivalente peut, quant à elle, laisser place à des sentiments de regrets et de rancunes qui peuvent compliquer le processus de deuil (Waskowic et Chartier, 2003 ; Worden, 1982; Glick, Weiss et Parkes, 1974).

\section{LIMITES}

Bien qu'une méthodologie rigoureuse ait été employée, cette étude comprend néanmoins des limites. Le nombre restreint de participants dans chacun des groupes limite la généralisation des résultats. Toutefois, étant donné les difficultés de recrutement liées à cette population, le nombre de participants est raisonnable. Plusieurs variables n'ont pas été analysées, il est donc possible que d'autres variables, comme le cumul d'événements de vie depuis le décès du conjoint, contribuent à augmenter la détresse, l'insatisfaction de vie et l'insatisfaction de couple. Une autre limite se situe au niveau de l'analyse rétrospective de la vie de couple. De fait, le décès du conjoint, qu'il soit par suicide ou par accident, peut amener certains endeuillés à moduler leurs réponses après coup, soit en créant un biais positif ou négatif lorsque les participants décrivent leur vie de couple avant le décès. Cependant, rien n'indique une convergence de biais positif ou de biais négatif chez un groupe ou l'autre.

En conclusion, nos résultats mettent en évidence l'importance de la satisfaction de vie et de couple dans le processus du deuil et génèrent différentes pistes d'intervention lors de psychothérapies de deuil.

Nos résultats montrent que certaines personnes endeuillées présentent davantage de détresse psychologique; il semble toutefois que cette détresse ne soit pas associée exclusivement à la nature du décès, mais que d'autres variables puissent contribuer à expliquer la difficulté du deuil. Ces résultats soulignent donc l'importance d'une investigation plus approfondie des variables influençant les difficultés du processus de deuil.

\section{Bibliographie}

BAILLARGEON, J., G. DUBOIS et N. MARINEAU. (1988). "Traduction française de l'Échelle d'ajustement dyadique», Canadian Journal of Behavioral Sciences, vol. 18, p. 25-30.

BARRACLOUGH, B., J. BUNCH, B. NELSON et P. SAINSBURY (1974). "A hundred cases of suicide: Clinical aspects », British Journal of Psychiatry, vol. 125, p. 355-373.

BARRETT, T.W. et T.B. SCOTT (1989). "Development of the Grief Experience Questionnaire", Suicide and Life-Threatening Behavior, vol. 19, p. 201-215.

BLAIS, M.R., R.J. VALLERAND, L.G. PELLETIER et N.M. BRIÈRE (1989). "L'échelle de satisfaction de vie: Validation canadienne-française du "Satisfaction with Life Scale" ", Revue canadienne des sciences du comportement, vol. 21, $\mathrm{n}^{\circ} 2$, p. 210-223, dans L. BOUFFARD et S. LAPIERRE, 1997, «La mesure du bonheur ", Revue québécoise de psychologie, vol. $18, \mathrm{n}^{\circ} 2$, p. 271-310.

BONANNO, G.A., K. BOERNER et C.B. WORTMAN (2008). «Trajectories of grieving ", dans M.S. STROEBE, H.S. HANSSON, E. STROEBE (dir.), Handbook of Bereavement Research and Practice, Washington, American Psychological Association, p. 287-307.

BOUFFARD, L., E. BASTIN et S. LAPIERRE (1997). Validation du PANAS (Positive affect and negative affect scales), manuscrit, Université de Sherbrooke, dans L. BOUFFARD et S. LAPIERRE, 1997, « La mesure du bonheur ", Revue québécoise de psychologie, vol. 18, $\mathrm{n}^{\circ} 2$, p. 271-310.

BOUTHILLIER, D., N. TREMBLAY, F. HAMELIN, D. JULIEN et P. SCHERZER (1996). "Traduction et validation canadienne-française d'un questionnaire évaluant l'attachement chez l'adulte", Revue canadienne des sciences du comportement, vol. $28, \mathrm{n}^{\circ} 1$, p. $74-77$.

BOZZINI, L. et R. TESSIER (1989). "L'insatisfaction maritale et l'intensité du deuil post-séparation: leurs effets sur la santé physique et le bien-être psychologique», Laboratoire de recherche en écologie humaine et sociale, UQAM, version préliminaire non publiée, dans J.L. HÉTU, 1994, Psychologie du mourir et du deuil, Montréal, Éditions du Méridien.

BRENT, D.A., J.A. PERPER et C.J. ALLMAN (1987). "Alcohol, firearms, and suicide among youth», Journal of the American Medical Association, vol. 257, p. 3369-3372.

BROWN, A.C., I.N. SANDLER, J.Y. TEIN, X. LIU et R.A. HAINE (2007). "Implications of parental suicide and violent death for promotion of resilience of parentallybereaved children », Death Studies, vol. 31, p. 301-335

CAVANAGH, J., A.J. CARSON, M. SHARPE et S.M. LAWRIE (2003). "Psychological autopsy studies of suicide: A systematic review », Psychological Medicine, vol. 33, p. 395-405

CEREL, J., M.A. FRISTAD, E.B. WELLER et R.A. WELLER (2000). «Suicide-bereaved children and adolescents: II. Parental and family functioning ", Journal of the American Academy of Child and Adolescent Psychiatry, vol. 39, p. 437-444.

CHENG, A.T., T.H. CHEN, C.C. CHEN et R. JENKINS (2000). «Psychosocial and psychiatric risk factors for suicide. Case-control psychological autopsy study», British Journal of Psychiatry, vol. 177, p. 360-365.

COLIN, V.L. (1996). Human Attachment, New York, McGraw-Hill.

CURRIER, J.M., J.M. HOLLAND et R.A. NEIMEYER (2006). «Sense-making, grief, and the experience of violent loss: Toward a meditational model », Death Studies, vol. 30, p. 403-428

CVINAR, J.G. (2005). "Do suicide survivors suffer social stigma: A review of the literature ", Perspectives in Psychiatric Care, vol. 41 , p. $14-21$.

DE GROOT, M.H., J. DE KEIJSER et J. NEELEMAN (2006). "Grief shortly after suicide and natural death: A comparative study among spouses and first-degree relatives ", Suicide and Life-Threatening Behavior, vol. 36, $\mathrm{n}^{\circ} 4$, p. 418-431.

DIENER, E., R.A. EMMONS, R.J. LARSEN et S. GRIFFIN (1985). "The Satisfaction with Life scale", Journal of Personality Assessment, vol. 49, p. 71-76.

DOMINGOS, B. et M.R. MALUF (2003) "Experiences of loss and mourning among school-children aged 13 to 18 ", Psicologia: Reflexao e Critica, vol. 16, p. 577-589.

DYREGROV, K., D. NORDANGER et A DYREGROV (2003). «Predictors of psychological distress after suicide, SIDS and accidents ", Death Studies, vol. 27, p. 143-165.

FEIGELMAN, W., J.R. JORDAN et B.S. GORMAN (2009). "How they died, time since loss, and bereavement outcomes", 
Omega: Journal of Death and Dying, vol. 58, p. 251-273.

FIELD, N.P. et E.C. SUNDIN (2001). "Attachment style in adjustment to conjugal bereavement», Journal of Social and Personal Relationships, vol. 18, p. 347-361.

FITZGERALD, E. (2005). "An investigation of traumatic grief, in suicide survivors and survivors of other types of death, in a Irish population », Dissertation Abstracts International: Section B: The Sciences and Engineering, vol. 66 (6-B), p. 3405.

FRALEY, R. et G.A. BONANNO (2004). «Attachment and loss: A test of three competing models on the association between attachment-related avoidance and adaptation to bereavement», Personality and Social Psychology Bulletin, vol. 30, p. 878890.

GASS, K.A. (1989). "Appraisal, coping, and resources: Markers associated with the health of aged widows and widowers ", dans D.A. LUND, 1989, Older Bereaved Spouses: Research with Practical Applications, New York, Hemisphere.

GLICK, I., R. WEISS et C.M. PARKES (1974). The First Year of Bereavement, New York, John Wiley and Sons.

HARWOOD, D.M.J., K. HAWTON, T. HOPE, L. HARRISS et R. JACOBY (2006).

"Life problems and physical illness as risk factors for suicide in older people: A descriptive and case-control study ", Psychological Medecine, vol. 36, p. 1265-1274.

HAWTON, K., S. SIMKIN et S. REES (2008). "Help at hand for people bereaved by suicide and other traumatic death ", Psychiatric Bulletin, vol. 32, p. 309-311.

HOUCK, J.A. (2007). "A comparaison on grief reactions in cancer, HIV/AIDS, and suicide bereavement », Journal of HIV/AIDS and Social Services, vol. 6, p. 97-112.

ILFELD, F.W. (1976). «Further validation of a psychiatric symptom index in a normal population ", Psychological Reports, vol. 39, $\mathrm{n}^{\circ}$ 3, p. 1215-1228.

JORDAN, J.R. (2008). «Bereavement after suicide », Psychiatric Annals, vol. 38, p. 679685.

JORDAN, J.R. et J. MCMENAMY (2004). "Interventions for suicide survivors: A review of literature», Suicide and Life-Threatening Behavior, vol. 34, p. 337349.

KALISH, R.A. (1981). Death, Grief and Mourning, Garden City, NY, Doubleday.

KIM, C.D., A.D. LESAGE, M. SÉGUIN, O. LIPP, C. VANIER et G. TURECKI (2003). "Patterns of co-morbidity in male suicide completer », Psychological Medicine, vol. 33, p. 1299-1309.

KITSON, G.C. (2000). "Adjustment to violent and natural deaths in later and earlier life for Black and White widows », Journals of Gerontology: Series B: Psychological Sciences and Social Sciences, vol. 55B, p. S341-S351.
KLINGER, E. (1977). Meaning and Void: Inner Experience and the Incentives in People's Lives, Minneapolis, University of Minnesota Press, XIV, 412 p.

LIEBSCHER, B.J. (2001). «Parental death and the impact on grieving children: A comparison of homicide or suicide and natural or accidental deaths », Dissertation Abstracts international: Section B: The Sciences and Engineering, vol. 62 (2-B), p. 1089.

LUND, D.A., M.S. CASERTA et M.F. DIMOND (1985). "The impact of spousal bereavement on the subjective well-being of older adults ", dans D.A. Lund, 1989, Older Bereaved Spouses: Research with Practical Applications, New York, Hemisphere.

MELHEM, N.M., G. MORITZ, M. WALKER, M.K. SHEAR et D. BRENT (2007). "Phenomenology and correlates of complicated grief in children and adolescents ", Journal of the American Academy of Child and Adolescent Psychiatry, vol. 46, p. 493-499.

MINISTÈRE DE LA SANTÉ ET DES SERVICES SOCIAUX DU QUÉBEC (2007). «Profil du suicide au Québec. 19812005p: mise à jour en $2007 »$, Service de la surveillance de l'état de santé, en ligne, <http://www.msss.gouv.qc.ca/sujets/ prob_sante/pdf/Profil-suicide-fev07.pdf $>$, consulté le 2010-10-12.

MITCHELL, A.M., D.D. GALE, L. GARAND et S. WESNER (2003). "The use of narrative data to inform the psychotherapeutic group process with suicide survivors", Issues in Mental Health Nursing, vol. 24, p. 91-106.

MITCHELL, A.M., S. WESNER, L. BROWNSON，D. DYSART-GALE，L. GARAND et A. HAVILL (2006). "Effective communication with bereaved child survivors of suicide ", Journal of Child and Adolescent Psychiatric Nursing, vol. 19, p. 130-136.

MURPHY, S.A., L.C. JOHNSON et J. LOHAN (2003). "Finding meaning in a child's violent death: A five-year prospective analysis of parents personal narratives and empirical data», Death Studies, vol. 27, p. 381-404.

MURPHY, S.A., L.C. JOHNSON, J. LOHAN et V.J. TAPPER (2002). «Bereaved parents use of individual, family, and community resources 4 to 60 months after a child's violent death », Family and Community Health, vol. 25 , p. $71-82$.

MURPHY, S.A., L.C. JOHNSON, L. WU, J.J. FAN ET J. LOHAN (2003). «Bereaved parents outcomes 4 to 60 months after their children's death by accident, suicide, or homicide: A comparative study demonstrating differences », Death Studies, vol. 27, p. 39-61.

NOLEN-HOEKSEMA, S. et J. MORROW (1993). "Effects of rumination and distraction on naturally occurring depressed mood», Cognition and Emotion, vol. 7, p. $561-570$.
PARKES, C.M. et R.S. WEISS (1983). Recovery from Bereavement, New York, Basic Books.

PRÉVILLE M., R. BOYER, L. POTVIN, C. PERRAULT et G. LEGARÉ (1992). La détresse psychologique: détermination de la fiabilité et de la validité de la mesure utilisée dans l'enquête Santé Québec, Enquête Santé Québec 87, Les cahiers de recherche, $\mathrm{n}^{\circ} 7$, Ministère de la Santé et des Services sociaux, $54 \mathrm{p}$.

RENGIFO, M.E. (2004). «Grief experiences in widows of policemen", Revista Latinoamericana de Psicologica, vol. $36, \mathrm{n}^{\circ} 1$, p. 33-46.

REQUARTH, M. (2006). After a Parent's Suicide: Helping Children Heal, Sebastopol, CA, Healing Hearts Press.

RICH, C.L., R.C. FOWLER, L.A. FOGARTY et D. YOUNG (1988). "San Diego suicide study: III. Relationships between diagnoses and stressors ", Archives of General Psychiatry, vol. 45, p. 589-592.

RICH, C.L., D. YOUNG et R.C. FOWLER (1986). "San Diego suicide study: I. Young vs old subjects ", Archives of General Psychiatry, vol. 43, p. 577-582.

SABOURIN, S., G. BOUCHARD, J. ERIGHT, Y. LUSSIER et C. BOUCHER (1988). "The influence of sex on the factorial invariance of the Dyadic Adjustment Scale», Science et comportement, vol. 18, p. 187-201.

SABOURIN, S., Y. LUSSIER et J. WRIGHT (1991). «The effects of measurement strategy on attributions for marital problems and behaviors", Journal of Applied Social Psychology, vol. 21, p. 734-746.

SCHAEFER, J. A. et R.H. MOOS (2001). "Bereavement experiences and personal growth", dans W. STROEBE, 2001, Handbook of Bereavement Research, Washington D.C., American Psychological Association.

SÉGUIN, M. (2009). Le suicide, le comprendre pour le prévenir, Boisbriand, Les éditions du Carré.

SÉGUIN, M., A. BRUNET ET L. LEBLANC (2006). Intervention en situation de crise et en contexte traumatique, Boucherville, Gaëtan Morin éditeur.

SÉGUIN, M. et L. FRÉCHETTE (1999). Le deuil une souffrance à comprendre pour mieux intervenir, Québec, Les éditions Logiques.

SETHI, S. et S.C. BHARGAVA (2003). "Child and adolescent survivors of suicide», CRISIS: The Journal of Crisis Intervention and Suicide Prevention, vol. 24, p. 4-6.

SIMPSON, J.A. (1990). "Influence of attachment styles on romantic relationships », Journal of Personality and Social Psychology, vol. 59, p. 971-980. 
SNOW, L. et M. MCHUGH (2002). "The aftermath of a death in prison custody", dans G. TOWL, L. SNOW et M. MCHUGH, 2002, Studies in Prisons, Malden, MA, Blackwell Publishing.

SPANIER, G.B. (1976). «Measuring dyadic adjustment: New scales for assessing the equality of marriage and similar dyads", Journal of Marriage and the Family, vol. 15, p. 15-28.

STEIN, N., S. FOLKMAN, T. TRABASSO et T.A. RICHARDS (1997) «Appraisal and goal processes as predictors of psychological well-being in bereaved caregivers ", Journal of Personality and Social Psychology, vol. $72, n^{\circ} 4$, p. 872-884.

STROEBE, M.S., H. SCHUT et W. STROEBE (2005). "Attachment in coping with bereavement: A theoretical integration ", Review of General Psychology, vol. 9, p. 48-66.

SVEEN, C.A. et F.A. WALBY (2008). «Suicide survivors mental health and grief reactions: A systematic review of controlled studies", Suicide and Life-Threatening Behavior, vol. 38, p. 13-29.
TALL, K., K. KAULVES, M. SISASK et A. VARNIK (2008). "Do survivors respond differently when alcohol abuse complicates suicide? Findings from the psychological autopsy study in Estonia », Drug and Alcohol Dependence, vol. 95, n 1-2, p. 129-133.

VALLERAND, R.J. (1989). "Vers une méthodologie de validation trans-culturelle de questionnaires psychologiques: Implications pour la recherche en langue française », Psychologie canadienne, vol. 30, $\mathrm{n}^{\circ}$ 4, p. 662-680.

VAN DOORN, C., S.V. KASL, L.C. BEERY, S.C. JACOBS et H.G. PRIGERSON (1998). "The influence of marital quality and attachment styles on traumatic grief and depressive symptoms ", Journal of Nervous and Mental Disease, vol. 186, p. 566-573.

WASKOWIC, T.D. et B.M. CHARTIER (2003). «Attachment and experience of grief following the loss of a spouse ", Omega: The Journal of Death and Dying, vol. 47, p. 77-91.

WATSON, D., A.L. CLARK et D. TELLEGEN (1988). "Development and validation of brief measures of positive and negative affect: The PANAS scales ", Journal of Personality and Social Psychology, vol. 54, p. 1063-1070.
WAYMENT, H.A. et J. VIERTHALER (2002). «Attachment style and bereavement reactions ", Journal of Loss and Trauma, vol. 7, p. 129-149.

WINTER, S., E. BROCKMANN et U. Hegerl (2005). "Experiences and needs of people bereaved by suicide», Verhaltenstherapie, vol. 15 , p. 47-53

WORDEN, J.W. (1982). Grief Counseling and Grief Therapy, New York, Springer Publishing Company.

WORTMAN, C.B. et R.C. SILVER (1989). "The myths of coping with loss», Journal of Consulting and Clinical Psychology, vol. 57, $\mathrm{n}^{\circ}$ 3, p. 349-357.

ZHANG, J., H.Q. TONG et L. ZHOU (2005). "The effect of bereavement due to suicide on survivors depression: A study of Chinese samples », Omega: Journal of Death and Dying, vol. 51, $\mathrm{n}^{\circ}$ 3, p. 217-227. 